\title{
Artificial Intelligence and its Application in Various Fields
}

\author{
Rohit Kumar Yadav, Munna Pandey
}

\begin{abstract}
The term 'AI' is not a new term but the actual meaning of ai is still hidden. Artificial intelligence is a branch of computer science that aims to create machines which are as intelligent as human beings. AI mainly focus on some questions like knowledge required while thinking, the way knowledge can be presented and the way knowledge can be used in other field's viz. Robotics. Scope of AI is much wider than our thinking. It is not limited to only one or two areas rather in coming future everything will be directly or indirectly linked to AI. Much research has been done on artificial intelligence which has shown that by the end of 2020 many works which was not possible by human beings will be efficiently and accurately can be carried out by the help of robots. Robotics is a branch of engineering that deals with formation, designing, manufacturing, operation of robots. Artificial intelligence is being applied to many areas which are capable to solve many problems like in robotics, e-commerce, domestic chores, medical treatment, gaming, mathematics, military planning etc. The main idea behind the merging of artificial intelligence and robotics is to optimize the level of autonomy through learning. In the coming future we can surely overcome the disadvantages of robots like misuse of it with the help of facial recognition. Or we can use AI in other fields like in cyber security to prevent the systems from being hacked. The applications of $\mathrm{AI}$ and how we can implement other applications in coming time are discussed adding to it how we can overcome the disadvantages of using robots in regular life are also discussed.
\end{abstract}

Keywords: Artificial intelligence, cyber security, face recognition, machines, robotics, robots,

\section{INTRODUCTION}

"I believe this artificial intelligence is going to be our partner. If we misuse it, it will be a risk. If we use it right, it can be our partner." -Masayoshi Son AI is the combination of different technologies that make the computer "think" and take decisions that help us to optimize tasks. One thing that makes us different from any other thing in the world is our intelligence. The ability that we have to apply knowledge , the ability to improve ourselves or the ability to understand are some factors that help in the evolution of civilization. "John McCarthy, an American scientist, popularly known as the father of Artificial Intelligence (AI) after playing crucial role in defining this area." He later devoted his time in the development of intelligent machines. "Artificial Intelligence is the branch of computer science that aims to create a robot controlled by computer or software that can think as intelligent as human beings." "Robotics is a branch of engineering that deals with formation, designing, manufacturing, operation of robots."

Revised Manuscript Received on June 30, 2020.

* Correspondence Author

Dr. Rohit Kumar Yadav*, Assistant Professor Department of Computer Science, IITM Janakpuri (GGSIPU) New Delhi. India.

Mr. Munna Pandey, Assistant Professor Department of Information Technology, IITM Janakpuri (GGSIPU) New Delhi. India.

(C) The Authors. Published by Blue Eyes Intelligence Engineering and Sciences Publication (BEIESP). This is an open access article under the CC BY-NC-ND license (http://creativecommons.org/licenses/by-nc$\underline{\mathrm{nd} / 4.0 /)}$
AI can be achieved by studying how human brain think, learn, study, decide, work while trying to overcome a problem and then successfully making use of this study as a basis of development of intelligent machines or software to control robots. AI is now being the talk of town and its scope is being increasing day by day. AI and robotics had hit all the emerging areas whether being like e-commerce, domestic chores, medical treatment, gaming, mathematics, military planning etc and now research is going on the other areas where AI can be used. There is endless variety in the size, shape, design of robot. Moreover there are lot of work and research going on the development and advancement of robots and artificial intelligence as we are sure of the bright future in the combination of robotics and AI. But our main focus lies in the area of future scope of Artificial intelligence and how to overcome on the various disadvantages of robots. So that the results of both the areas are efficient, reliable and accurate.

A) Goals of AI

There are mainly two goals of AI.

1. Create Expert systems: - These systems are the system which portrays intelligent behavior in terms of learning, demonstrate and to advice its users.

2. Implementing Human Intelligence: - These types of systems which can understand think learn or behave just like human beings.

\section{ROBOTS CONTROL SYSTEM}

The task of robot is to sequentially execute the planned motion. The expected change in each parameter is calculated and the actuator energy which is needed to achieve that change is calculated and the energy which is achieved is applied to the actuator. If the model designed is correct then no disturbances will be faced and the expected change will be achieved.

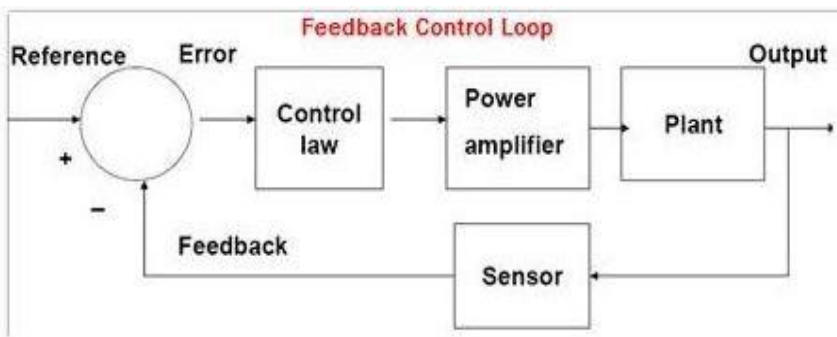

Feedback Forward loop is used to determine the speed from one sensor to another. The position of a robot arm is seen by the position sensor and power of actuators is altered so that movement confirms the desired path. Feed forward control is used to predict how much action should be taken or how much energy to be used.
Blue Eyes Intelligence Engineering \& Sciences Publication (C) Copyriaht: All riahts reserved.

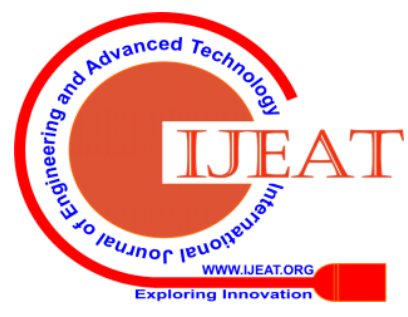




\section{Artificial Intelligence and its Application in Various Fields}

\section{HOW ROBOTS MAKE DECISION?}

Robots make decision using Boolean algebra where they need to take more than one conditions in to their minds which can be combined using logical operators and they make decisions based upon the result of the conditions.

The resulting values are known as the truth values. These truth values tell robots whether the condition is true or false.

\section{A) BRANCHING:-}

The idea of breaking the condition into truth values, robots make their task complete based upon the result. The idea of performing task based upon the result is known as branching.

Example:-

For instance, a robot that has an Ultrasonic sensor attached to it. If we want robot to drive a car without hitting anybody and to follow traffic rules then we can give robots certain rules like:-

Task main ()

\section{\{}

While (condition)

\section{\{}

If (condition)

\{// commands $\}$

Else

\{// commands $\}$

\}

\}

\section{B) Can we create ethical robots?}

As robots are becoming more developed and advanced then their ethical decision making capability will become more innovatory.

There are two main approaches to create ethical robots:-

1. The first is to write an ethical law and create a robot that strictly follows the code. But the difficulty is to decide an appropriate ethical law.

2. The second approach is to create a learning robot and to teach the robot how to respond to a particular situation. This is much similar to how humans learn things or values but the query over here is that are humans itself the best morality teachers?

\section{C) Locomotion and Manipulation}

Robots are systems that can sense, actuate, compute, and communicate just like human beings.

Locomotion: - It is defined as the ability to move the robot. Locomotion includes very different concepts of motions like crawling, swimming, flying, walking, sliding etc.

Manipulation: - It means ability to move object with respect of robot.

D) Can robots be a substitute of humans?

Many reports claimed that major part of human force will be replaced machines in coming years. With many research being conducted on artificial intelligence has shown that there will be major job crisis because multiple jobs at a time can be efficiently and accurately be carried out by the utilization of a single machine.

But the question arises is it really possible for machine to completely replace human era?

We all completely agree that human can be replaced by machines but the fact is that it is impossible for a machine to completely replace humans. As long as AI is not evolved to a point of developed machines which can interact, learn, think, respond, solve just like human beings it's not possible for intelligent machines to completely replace the human resource. We all can surely say that in the coming years robots cannot replace human beings but the thing we can see is that the workforce will be a combination of human beings and robots so that all the records and workload can be managed efficiently and precisely. We are moving towards future where we can see human and robots working together more closely than ever expected.

\section{ARTIFICIAL INTELLIGENCE AND ROBOTICS ARE NOT SAME}

The first thing that should be clear is that artificial intelligence and robotics are not the same.

Robots are hardware whereas artificial intelligence is software. For robotics we need to study mechanical engineering and for artificial intelligence we need to study computer science. There is a combination of robotics and AI in the form of learning robots. Robots should be as intelligent as possible to be effective, so they need AI. But AI is practiced on ordinary computers more often than on robots.

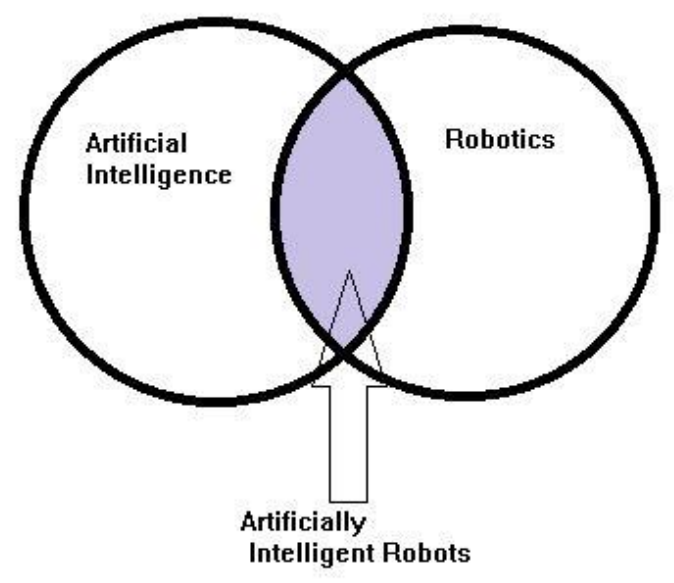

\section{APPLICATIONS OF ARTIFICIAL INTELLIGENCE}

The most appreciating and well known applications of AI like Netflix, spottily and siri and many other are present today but through this much more vast applications of AI in different fields like of robotics, medical, social and technology will be discussed.

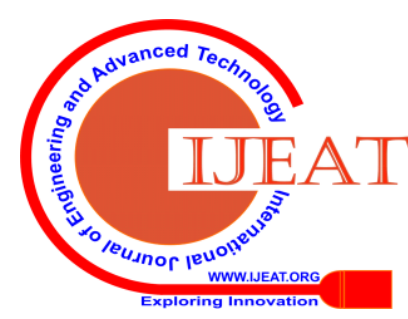




\section{Robotics:}

Once a mechanical robot is built, it cannot work by itself but also needs a way to think, act and work upon feelings just like humans being have and that is what AI provides to the field of robotics.

I-robots are not only confined with their work in industries, medicals and in the field of exploration but also have contributed its growth in the entertainment field as well. Ex: the movie named wall -e and robot etc.

\section{Medical domain:}

a) Assistive communication: AI researches are constantly working not only for the living mass but also for the mass who is not able to speak, hear or even see and this assistive communication can be achieved using AI which will help them (the people suffering from any severe disease) to think of any alphabet or any word which then can be seen on a computer screen using the body sensors. This can be achieved using some electrodes connected to the brain which can sense what the patient is trying to tell and decode that with the help of different algorithms and encoding schemes and then can represent the result on the virtual screen connected. This type of research program will not only be a big success but also a start a new era for the people with adverse neurological diseases.

b) Automated hand glove: Using AI one can communicate their thoughts and works with their respective robots to do the desired work on behalf of them so as to save the time and the human labor. It does not matter where the human being is but through any device like a watch or a band can be used as a medium to communicate with each other (human and its robot).

c) Developing molecularly similar robots: through $\mathrm{AI}$ even the molecular robots having the replicated DNA of any individual can also be carried out.

d) Super Intelligent Doctors: Say No more to deaths and yes to the cure. Using AI and deep learning robots can perform surgeries or researches if needed in future times as well.

e) Fuzzy expert system in medicines: Till today the diagnosis of leukemia and breast cancer can only be resolved using fuzzy logics. Fuzzy logic is basically an approach to get the computerized "true result" of something in the form of true and false. A fuzzy logic-based expert system for diagnostic decision support is presented (MEDUSA). The representation of the uncertain and imprecise knowledge about things like this is realized by fuzzy sets the desired relations.

\section{Spatial Domain:}

Does life exist on other planet? Or who doesn't want to see what's there out of this planet Earth?

Ask AI and it will provide you with all the basic information about it even with the less specific details.

A single individual can't survive in many fatal conditions like if it is a new planet or a new place (because of low humidity, no gravity, no water etc) but a robot (a machine) can. Using this new intelligence big companies like NASA and ISRO are constantly working to give out new missiles and rockets for the betterment of the country

Ex: like using this NASA is even preparing one such robot for its mission "mars 2020".

\section{Domestic and Social Domain:}

a)A big helping hand: According to a Hollywood movie name 'I-robot' and a famous cartoon 'Richie rich' suggested that even machines can help people do their house hold chores with much precision and less time

b) Self -running cars: "In a survey ${ }^{[1]}$ every day in America, another 29 people die as a result of drunken driving crashes. That's one person every 50 minutes."

This thing can easily be minimized or even stopped by using AI. As soon as the car senses that the driver has lost its control and can't drive the car ,reasons can be anything via drinking, ill health or mental stress, it will automatically activate the cars self driving and will leave the person to the location specified by him or her at the very first time.

\section{Life Domain:}

Can AI be a life saver too??

Well the answer to the above question is yes it can be, how let's talk about that.

a)Radiation and Mine work: People working in underground mines and at places having high amount of radiation have huge amount of ill effects on their health and family as well so AI provides a good and improved of using specially made robots to work there without having any ill effects of that to them.

b) Using $\mathrm{AI}$ in the time of a natural calamity: At the time of flood or biggers natural calamities, the specialized type of robotics can be used so as to help people to be safe from the adverse effects.

c) Army Soldiers: According to a censor as of July 27, 2018, there have been 2,372 U.S. military deaths in the War in Afghanistan. 1,856 of these deaths have been the result of hostile action. 20,320 American service members have also been wounded in action during the war. ${ }^{[2]}$

To stop the unnecessary killing of people just to acquire the land or prosperity, now human can use robots on the battle fields so as to have a battle in which no human loss is prevailed.

${ }^{[2]}$ And not the robots using AI can be developed to help saving the mankind but even the high tech machines to select the target and the missile launching can also be done using IDS (intrusion detection system) of AI. Intrusion Detection System is a process which uses various AI techniques to securing the data from the intruders

\section{6) AI used in cyber defense:}

Now days many social networking sites are available for people to communicate with each other but with this comes a major role of each individual security, security of their data and their privacy from the outside world. When somebody logs in two types of password is generated one which user enters and the one which server enters and the other one is all in encrypted form which cannot be accessed by any other person and $\mathrm{AI}$ is a big technique of letting this come into action.

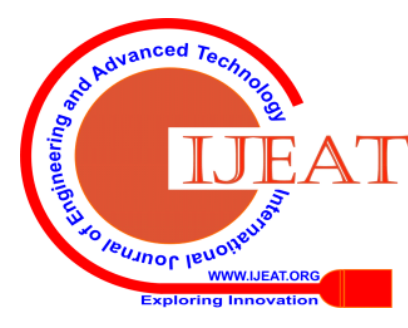




\section{Artificial Intelligence and its Application in Various Fields}

7) Physical biometric solution, use of facial or eye recognition [3]:

Biometric solutions are of two types namely: physical and behavioral and this type of face and eye recognition comes under the physical biometric solutions and this is a much demanding topic not only in robots but also in electronic devices like computer, smart phones and laptops as well. These days robots which were once made for the betterment of the people can easily be used for wrong deeds .And once the mechanism of robot get changed ,it will cause destruction like never thought of so as to stop this to happen in future a process called face-recognition system is used in which the system identifies, verifies and then compares the selected facial features with the digital image stored in its database at the time of its creation for ex: specific algorithms are used to analyze different features like distance between eyes, shape of nose, the lower jaw spacing and many more ,I-robots save the face patterns of its maker as an authorized person and then compare it with the eye patterns of the unauthorized person so as to save itself from being a cause of the mass destruction. It's true that technology advances by leaps and bounds and we grow more the AI will be growing more with us.

\section{PROS AND CONS IN THE MERGER OF ARTIFICIAL INTELLIGENCE AND ROBOTICS}

Everything which we see in this world is two faced. If we are talking about the advantages and applications then for sure it will have some disadvantages too. But there is nothing in this world which cannot be done. It is possible that we can surely overcome many of disadvantages with the help of some technologies.

Advantages:-

1. Error Reduction: - With the help of robots we can increase the quality of work. The results will be more efficient and accurate.

2. Force Multiplier: - With the help of advancement in technology we can see that robotics can increase the potential of team.

3. Reduce Risk For Humans: - With the help of robots we can accomplish all the tasks which seem to be harmful for human beings.

4. Incorrupt: - As robotics follows on the commands given by human beings we can say that robots are incorrupt which cannot fold their eyes.

Disadvantages:-

1. Misuse of robots: - Robots parts can be easily misused. Way of overcoming: - We can use biometrics so that only authorized person can access the part of robots.

2. Create Unemployment: - With the use of robots in every field human era can be replaced.

Way of overcoming: - We can make our workers skilled by teaching how they can create mini robots so that they can also contribute in the advancement of country.

\section{CONCLUSION}

This paper covers the basic aspects and the wide range in which the AI is used, being used or will be using in future. And because of its tremendous growth AI is one the most trending topic on which much of research is already done and some of it is still going on. Technique like Intrusion
Detection System has a wide area of future analysis. We conclude that if not done well then AI can be programmed to do something devastating or can be programmed to do the good for the people and this depends on only us, "the humans" so think wise and use each and every technique of all $\mathrm{AI}$ in a wise way so as to make AI and its applications a far reaching effects on human life in the upcoming years.

\section{REFERENCES}

1. Carrot.whitman.edu. (2018). [online] Available at: https://carrot.whitman.edu/Robots/PDF/Decision\%20Making.pdf [Accessed 19 Aug. 2018.

2. Quartz. (2018). Can we trust robots to make moral decisions? [online] Available at: https:/qz.com/653575/can-we-trust-robots-tomake-moral-decisions/ [Accessed 19 Aug. 2018].

3. Pdfs.semanticscholar.org. (2018). [online] Available at https://pdfs.semanticscholar.org/450c/dd4d7650e457544454996f47d 677fe45f28a.pdf [Accessed 19 Aug. 2018].

4. Correll, N. (2018). Introduction to Robotics \#2: Locomotion and Manipulation. [online] Correll Lab. Available at: http://correll.cs.colorado.edu/?p=869 [Accessed 19 Aug. 2018].

5. Medium. (2018). Artificial Intelligence: Can it Replace Human Intelligence?. [online] Available at: https://medium.com/@BrentMorgan/artificial-intelligence-can-itreplace-human-intelligence-b250b9aec153 [Accessed 19 Aug. 2018].

6. Owen-Hill, A. (2018). What's the Difference Between Robotics and Artificial Intelligence?. [online] Blog.robotiq.com. Available at: https://blog.robotiq.com/whats-the-difference-between-robotics-andartificial-intelligence [Accessed 19 Aug. 2018]. ${ }^{\text {[1] }}$

7. Statistics | MADD. (2018). Retrieved from https://www.madd.org/statistics/

8. https://en.wikipedia.org/wiki/United_States_military_casualties_in_t he_War_in_Afghanistan[3] https://www.techemergence.com/AI-inbiometrics-current-business-applications/

\section{AUTHOR PROFILE}

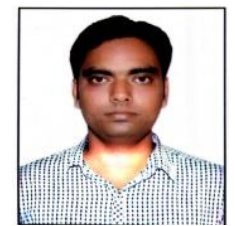

Dr. Rohit Kumar Yadav working as an Assistant Professor in the department of Computer Science at IITM Janakpuri (GGSIPU) New Delhi since july 2017. He has completed his M.sc from IIT Dhanbad in 2010. After that he had worked as a adhoc lecturer in government engineering college for one years. He has also completed his $\mathrm{Ph} . \mathrm{D}$ in computer science and engineering in 2017 from IIT Dhanbad. He has published more than 15 paper in international conference and repute journals (sci and scopus index). He is also reviewer and editor of some international conferences and reputed journals.

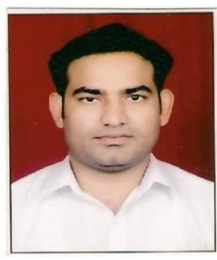

Mr. Munna Pandey working as an Assistant Professor in the department of Information Technology at IITM Janakpuri (GGSIPU) New Delhi since july 2009. He has completed his MCA from AIT Bangalore in 2007. After that he had worked as a lecturer in Lingaya's engineering college for one year. He is pursuing his Ph.D From Jiwaji University Gwalior (MP).

Blue Eyes Intelligence Engineering

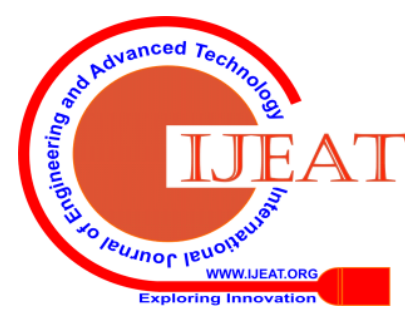

